\title{
Three-stub quarter wave superconducting resonator design
}

\author{
N. R. Lobanov and D. C. Weisser \\ Department of Nuclear Physics, Research School of Physical Sciences and Engineering, Australian National University, \\ Canberra, ACT 0200, Australia
}

(Received 3 July 2006; published 16 November 2006)

\begin{abstract}
This paper describes a concept for superconducting resonators for the acceleration of ions in the velocity range $\beta=v / c=0.015-0.04$. Such a resonator operates in $\lambda / 4$ mode with three loading elements and so can be thought of as a triple quarter wave resonator (3-QWR) providing 4 accelerating gaps. The use of a column to support the three stubs provides a benefit beyond those of the two-stub design (2-QWR). In the 3-QWR, the rf mirror currents in the walls surrounding the stubs need only travel through $45^{\circ}$ instead of the $90^{\circ}$ in the 2-QWR thus further reducing the current in the demountable joints. As in the 2-QWR, the shape of the column allows control of the frequency splitting between the accelerating and other modes. The copper structure is designed to be coated by a thin superconducting film of niobium or lead for operation at $4.3 \mathrm{~K}$. The particular device reported here operates at $150 \mathrm{MHz}$ with an optimum $\beta$ of 0.04. Its outer cylinder is the same size and shape as for the 2-QWR structure reported previously, in order to minimize construction and cryostat costs. A simple transmission line model is presented and the results of microwave studio and other numerical analyses are discussed. The 3-QWR resonators are appropriate for the upgrade of the low-velocity sections of the ANU Heavy Ion Accelerator Facility and other heavy ion accelerator boosters.
\end{abstract}

DOI: 10.1103/PhysRevSTAB.9.112002

PACS numbers: 29.17.+w, 29.27.-a, 41.75.-i

\section{INTRODUCTION}

Many heavy ion accelerators worldwide exploit superconducting rf and look to multigap structures in order to minimize infrastructure costs in catering for low-velocity ions. A successful solution to this problem has been the 4gap, interdigital quarter wave resonator for the velocity range 0.008 to $0.04 \beta$ [1] developed at Argonne National Lab for the Atlas injector. Three-gap resonators cover the somewhat higher, $\beta=0.04$ to 0.12 . The interdigital solution intrinsically has high currents between the rf stub and the surrounding coaxial cylinder and so requires high performance demountable joints between them or a fully welded construction. The rf joints, in spite of their careful design and robust engineering, are still suspected of dominating the resonators' rf losses. Neither the superconducting joints nor the fully welded options were appropriate to available resources here. Instead of following the single stub, quarter wave resonator path, we have explored the extension of 2-QWR, which has 3 accelerating gaps, to a 4-gap, 3-stub structure. It is not obvious that a 3-stub device has an eigenstate that provided a coordinated accelerating field in all four gaps. A model was built that indeed displayed such an eigenstate and this was confirmed with microwave studio (MWS) simulations.

A previous paper [2] presented a concept of $\lambda / 4$, 3-gap structure with two loading elements of a new design, the 2-QWR, for the velocity range $\beta=0.04-0.12$. The present paper discusses a $150 \mathrm{MHz} \lambda / 4$, 4-gap device with three loading elements (3-QWR) for optimum velocity $\beta=$ 0.04. The 3-QWR concept can be used for the velocity range $\beta=0.015-0.04$. Both the 2-QWR and 3-QWR are members of a family of TEM-like $\lambda / 4$ and $\lambda / 2$ structures terminated in the capacitive load of a drift tube and with multiple loading elements or stubs. Their combined lowvelocity range of $0.015<\beta<0.12$, recommend them as candidates for the front ends of LINACs intended as boosters for heavy ion electrostatic accelerators and as drivers for rare-isotope accelerators (RIA). The higher particle velocities of $0.15<\beta<0.6$ [3] are catered for by existing, standard superconducting cavities.

A $\beta<0.04$ accelerating structure can be achieved by either operating it at low frequency, which requires long loading elements, and/or employing short drift tubes and short gaps between them and higher operating frequencies. Both choices conflict with other crucial requirements. For example, long loading elements are flexible, exhibiting low frequency principal mechanical modes, which are readily excited by local vibrations. If the choice is made to use a high radio frequency with short drift tubes and accelerating gaps, then the beam apertures must also be small to maintain efficient coupling of the field to the beam. Small gaps create large capacitances that vary with small distance changes caused by mechanical vibrations. These gap length changes lead to rf resonant frequency variations, which require expensive countermeasures in the resonator control system.

The center-gap-to-center-gap distance in an accelerating structure is $d \approx(\beta \times \lambda) / 2$, where $\lambda$ is the $\mathrm{rf}$ wavelength. The $\beta<0.04$ structure requires $d<4 \mathrm{~cm}$ at an operating frequency of $150 \mathrm{MHz}$ and therefore the acceleration length of the 2-QWR is small. The 3-QWR, however, provides $50 \%$ more acceleration length for similar investments in electronics, cryogenics, and laboratory space but has two intrinsic disadvantages. First, it is less efficient for 
accelerating even $\beta$ matched beams because its transit time factor is $\sim 0.88$ compared to $\sim 0.90$ [4] for the 2-QWR. Second, the range of $\beta / \beta_{0}$, where $\beta_{0}$ is the optimum, for which the transit time factor is at least $60 \%$ of its maximum is $61 \%$ for the 3-QWR compared to $85 \%$ for the $2-\mathrm{QWR}$ and $158 \%$ for the 1-QWR based on the normalized transit time factors for the 2-, 3-, and 4-gap accelerating structures [5].

The $\beta$ mismatch problem for multigap resonators requires more design types for an accelerator than would be the case for resonators with fewer gaps. This burden is more than compensated by the 3-QWR's advantages.

\section{THREE-STUB RESONATOR: 3-QWR}

This report expands $\lambda / 4$ the twin resonator concept by introducing the three-stub $\lambda / 4$ resonator, 3-QWR. Similar to the 2-QWR, the 3-QWR incorporates a supporting column that controls the eigenfrequency splitting of now three fundamental modes. An added benefit of the 3-QWR over the 2-QWR is that the current in the demountable joints almost a factor of 2 lower. The demountable flanges facilitate fabrication and coating so that electron beam welding and vacuum brazing are not required.

The existence of the mode with acceleration field in all four gaps, the frequency splitting, and the small magnetic field at the demountable flanges have been confirmed using MWS simulations and experimentally in full-scale models. The 3-QWR also benefits from a central stub design that simultaneously achieves a uniform electrical field along the acceleration axis and balanced electric forces on the outer loading elements as described in Sec. IIE2.

\section{A. Transmission line approximation}

The gap lengths, $g$ in the 3-QWR, are in the ratio 1:2:2:1 as seen in Fig. 1. All the electric fields are equal because the voltage differences are $2 V_{0}$ across the $2 g$ gaps and $V_{0}$ across the $1 g$ gaps, where $\pm V_{0}$ is the peak voltage generated by the loading elements. Therefore the maximum energy gain is $\Delta E=6 q V_{0} T_{\beta} \cos \left(-18^{\circ}\right)$ for an ion of velocity $\beta$ in charge state $q$, where $T_{\beta}=0.88$ is the maximum transit time factor for 4 gap structure [5] and $-18^{\circ}$ is the phase. The accelerating field is $E_{\text {acc }}=6 V_{0} / L$, where $L=l+2 r_{a}=15.6 \mathrm{~cm}$ is the beam path length.

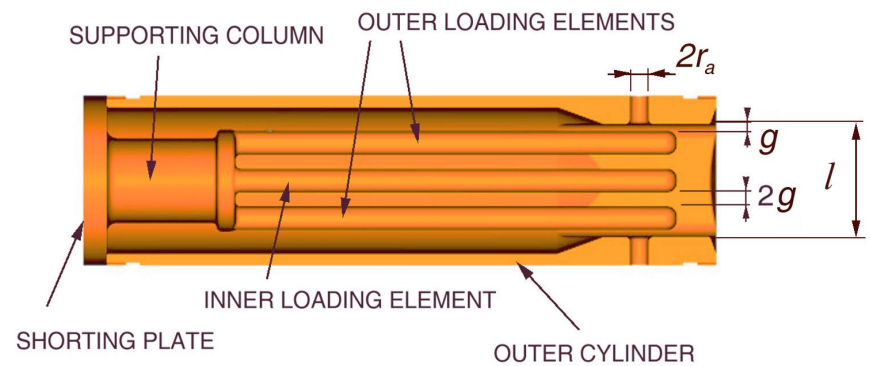

FIG. 1. (Color) A $150 \mathrm{MHz}$ 3-QWR for $\beta_{0}=0.04$.
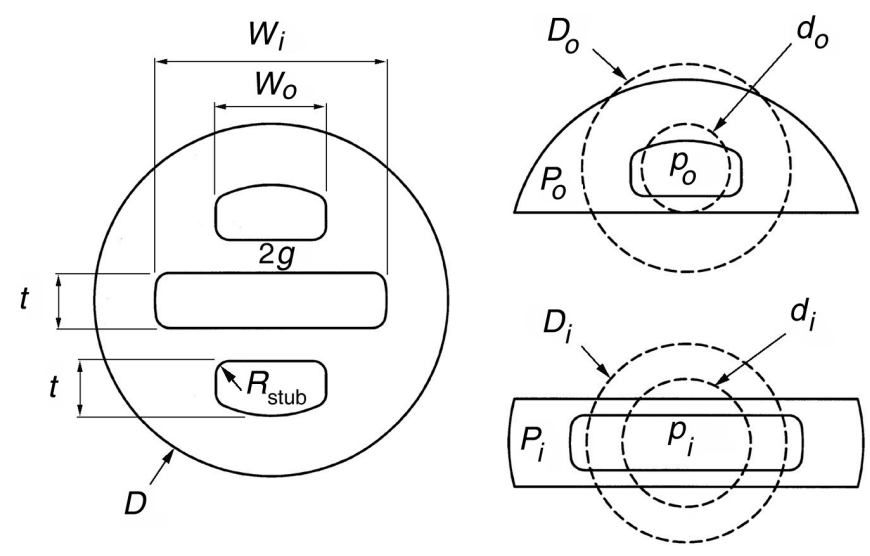

FIG. 2. The cross section of the 3-QWR and the equivalent coaxial transmission line for each loading element. The perimeters of the outer conductor and the loading element are $P_{i, o}$ and $p_{i, o}$, respectively.

The cavity physical length along beam axis is $l=14 \mathrm{~cm}$ and $r_{a}=0.8 \mathrm{~cm}$ is the radius of beam aperture.

The equivalent coaxial transmission line for each individual stub of the 3-QWR can be approximated to facilitate the application of the coaxial transmission line formulas [4]. The diameters of the outer and inner equivalent cylinders $D_{o}, D_{i}$ and the loading elements $d_{o}, d_{i}$, shown in Fig. 2, are determined by noting that the symmetry plane between a pair of stubs is an equipotential surface and so can be replaced by a ground plane. For the purposes of this approximation, the diameter for an equivalent coaxial electrode is taken to have the same circumference as the perimeter of the actual coaxial element i.e., $P_{i, o}=\pi D_{i, o}$ or $p_{i, o}=\pi d_{i, o}$, as illustrated in Fig. 2.

\section{B. Inner electrode-equivalent coaxial approximation}

Based on this approximation, the diameter of the outer equivalent conductor associated with the inner loading element is

$$
D_{i}=2\left\{\left(D^{2}-4 x^{2}\right)^{1 / 2}+D \arcsin (2 x / D)\right\} / \pi,
$$

where $x=g+t / 2$ is the midgap to centerline distance. Assuming that the stub cross section is rectangular, the inner equivalent conductor corresponds to a diameter $d_{i}=$ $2\left(w_{i}+t\right) / \pi$. For $D=16 \mathrm{~cm}, x=4 \mathrm{~cm}, W_{i}=10.4 \mathrm{~cm}$, and $t=2.5 \mathrm{~cm}$, the calculated diameters are $D_{i}=$ $12.4 \mathrm{~cm}$ and $d_{i}=8.2 \mathrm{~cm}$. The characteristic impedance of the equivalent coaxial transmission line is $Z_{i}=24.8 \Omega$.

C. Outer electrode-equivalent coaxial approximation

For the outer loading elements, the outer equivalent conductor's diameter can be taken to be

$$
\begin{aligned}
D_{o} & =0.5 D+\left\{\left(D^{2}-4 x^{2}\right)^{1 / 2}-D \arcsin (2 x / D\} / \pi\right. \\
& =11.6 \mathrm{~cm} .
\end{aligned}
$$


The diameter of the equivalent stub is $d_{o}=2\left(w_{o}+\right.$ $t) / \pi=4.8 \mathrm{~cm}$ for $w_{0}=5 \mathrm{~cm}$. The characteristic impedance $Z_{o}=52.9 \mathrm{~W}$ of the outer loading element is about twice that of the inner one. The maximum current at the shorted end through the outer loading element is half of that through the inner one. Thus, the rf current density in all loading elements is nearly the same since the crosssectional area of the inner element is about twice than that of the outer ones. Both outer and inner elements have the same physical length for $C_{l o} \approx 0.5 C_{l i} \approx 5 \mathrm{pF}$. The fulfillment of these conditions is sufficient to achieve balanced voltages generated by all loading elements, which results in uniform $E$-fields along the accelerating path. This simple coaxial transmission line approximation is in good agreement with MWS numerical simulations, as is discussed in the following section.

\section{Design optimization with microwave studio (MWS) [6]}

The 3-QWR has three principal low frequency rf eigenmodes. The first, mode 1 , is a nonoptimal mode in which current oscillates along all three loading elements in parallel and then to the outer cylinder, as shown in Fig. 3.

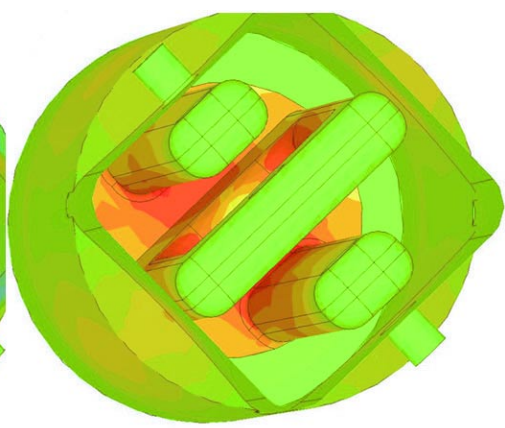

$H$-field

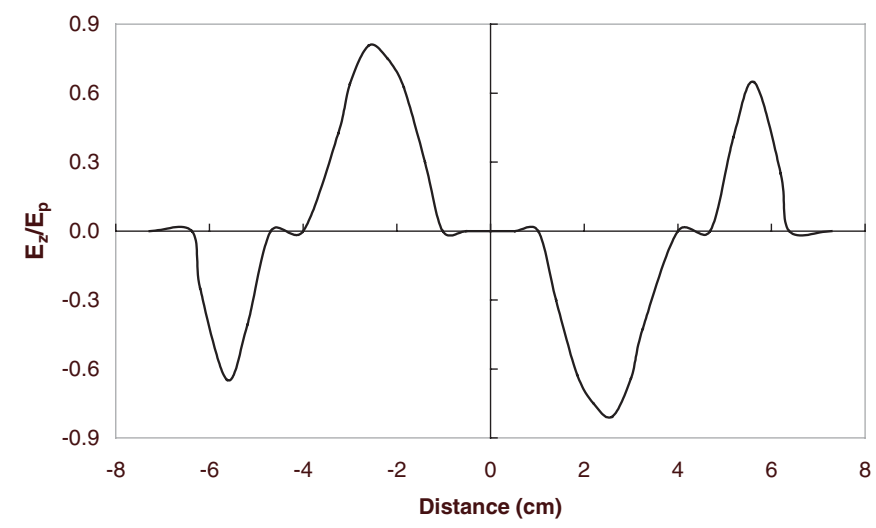
$E_{p}$ obtained with MWS, as a function of the distance along the beam $z$-axis in a 3-QWR. The zero of the $z$-axis is at the center of the cavity.

FIG. 4. (Color) Nonoptimal mode 2 for $E$-field and $H$-field in a 3-QWR. Blue represents the opposite polarity to red.

and the walls. Mode 2 is analogous to a $2-Q W R$ in which current oscillates between the two outer loading elements illustrated in Fig. 4. This is also an undesirable mode because there is only $V_{o}$ rather than $2 V_{o}$ in the gaps to the inner stub. Mode 3 is the desired accelerating mode in which current oscillates along all 3 loading elements from both outer ones in parallel and then to the inner one, as both sides of the central stub.

The frequency of mode 1 is lower than the (accelerating) length of the supporting column. The frequency of mode 2 is lower than that of the accelerating mode due to the added because between the bases of the outer loading elements frequency splitting is controlled by the geometry of the column supporting the stubs. The mode splitting of few $\mathrm{MHz}$ is large compared to the $\sim 100 \mathrm{~Hz}$ bandwidth of the overcoupled superconducting cavity and thus the accelerating mode can be selected uniquely. Mode 1 is analogous to a 1 -QWR with a high $H$-field, and therefore high current, in the shorting plate. There is no field between the stubs and only $V_{o}$ between the outer stubs

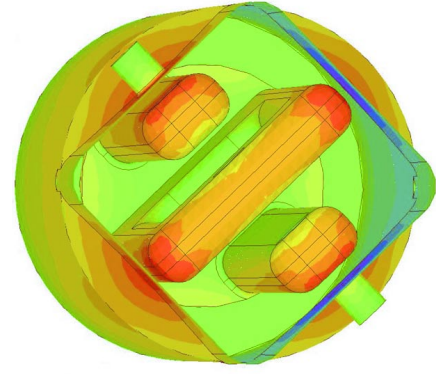

E- field

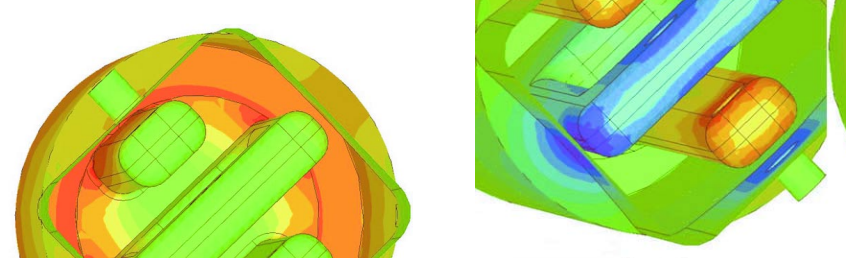

E- field
FIG. 5. (Color) Accelerating mode 3 for $E$-field and $H$-field in a 3-QWR. Blue represents the opposite polarity to red.

FIG. 6. The ratio of the accelerating field, $E_{z}$, to the peak field,

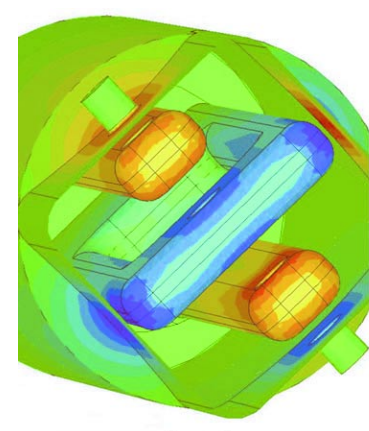

FIG. 3. (Color) Nonoptimal mode 1 for $E$-field and $H$-field in a 3-QWR. Red represents high values of fields of the same polarity and green, low fields.
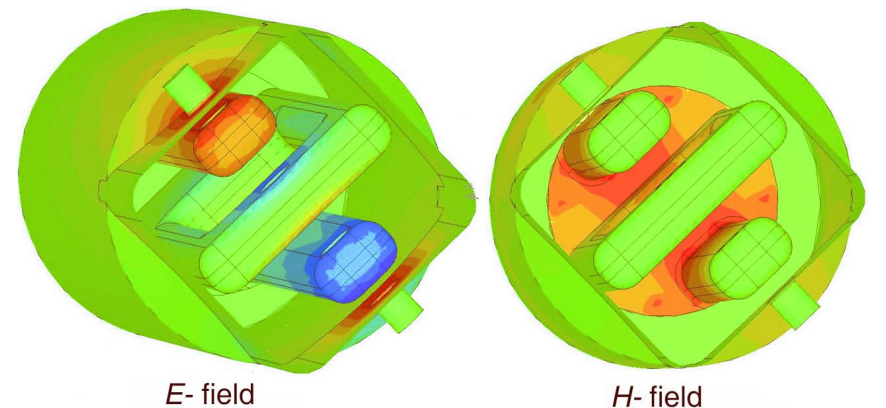

$H$ - field 
TABLE I. 3-QWR, rf parameters.

\begin{tabular}{ll}
\hline \hline Mode 3 frequency at $300 \mathrm{~K}$ & $150.428 \mathrm{MHz}$ \\
Mode 2 frequency at $300 \mathrm{~K}$ & $140.993 \mathrm{MHz}$ \\
Mode 1 frequency at $300 \mathrm{~K}$ & $104.683 \mathrm{MHz}$ \\
$\beta_{\text {opt }}$ & 0.04 \\
Beam path length $l+2 r_{a}$ & $15.6 \mathrm{~cm}$ \\
Energy gain $\Delta E / q$ at $E_{\mathrm{acc}}=1 \mathrm{MV} / \mathrm{m}$ and $\beta_{\mathrm{opt}}$ & $0.13 \mathrm{MV}$ \\
$E_{p} / E_{\mathrm{acc}}$ & 6.0 \\
$B_{p} / E_{\text {acc }}$ & $18.2 \mathrm{mT} /(\mathrm{MV} / \mathrm{m})$ \\
Stored energy, at $E_{\text {acc }}=1 \mathrm{MV} / \mathrm{m}$ & $46.1 \mathrm{~mJ} /(\mathrm{MV} / \mathrm{m})^{2}$ \\
$E_{\text {acc }}$ at Joule stored energy & $4.7 \mathrm{MV} / \mathrm{m}$ \\
$\Gamma=Q R_{s}$ & $12 \Omega$ \\
\hline \hline
\end{tabular}

The peak surface electric field, $E_{p}$, occurs at the end of each stub and depends on the end curvature and the stub edge radius, $R_{\text {stub }}$. The relative accelerating field profile $E_{z}$ along the beam axis is shown in Fig. 6 as calculated with MWS. The accelerating field, $E_{\text {acc }}$ is obtained by integrating $E_{z}$ resulting in $E_{p} / E_{\text {acc }}=6.0$ for the 3-QWR, not as good as the 4.5 to 5.0 for $\lambda / 4$ structures, Ref. [7].

The peak magnetic field $H_{p}$, thus the peak current, occurs on the internal surface where the loading elements meet the supporting column. The parameter effective in minimizing $H_{p}$ is $R_{\text {stub }}$, shown in Fig. 2. A value for $H_{p} / E_{\text {acc }}$ of $18.2 \mathrm{mT} / \mathrm{MV} / \mathrm{m}$ was calculated with the MWS simulation code for $R_{\text {stub }}=12 \mathrm{~mm}$. The results of MWS numerical simulations for the 3-QWR are summarized in Table I.

The perturbation method was used to calculate the quality factor, $Q$, of the copper cavity at $20^{\circ} \mathrm{C}$. The geometrical factor $\Gamma$, which depends only on the shape of the structure, is the product of $Q$ and the surface resistance $R_{s}$. The surface resistance is $R_{s}=\left(\pi f \mu_{0} \rho\right)^{1 / 2}=3.2 \mathrm{~m} \Omega$, where $f=150 \mathrm{MH} z, \quad \mu_{0}=4 \pi \times 10^{-7}, \quad$ and $\rho=$ $1.7 \times 10^{-8}(\Omega \times \mathrm{m})$ is the bulk resistivity of copper.

\section{E. Structural Properties}

The major contributor to amplitude and phase jitter of the resonator is changes of the spacing of the stub ends and, therefore, the eigenfrequency. These changes are caused by both ponderomotive effects and by mechanical vibrations due to vacuum pumps, refrigerator compressors, and from boil-off gas pressure variations. Variations in the electric field amplitude and phase from all causes are addressed by the rf control system.

\section{Microphonic and ponderomotive effects}

The resonator should be as stiff as possible to minimize the power needed in the control system to maintain the precise frequency and phase. Stiff structures are characterized by high frequency of the lowest principal mechanical modes. Since environmental vibrations are usually low frequency, it is desirable to have the lowest structural principal mode frequency well above the environmental ones. A stiff structure accomplishes both goals.

The ponderomotive effects become important [8] with increasing accelerating field $E_{\text {acc }}$. In $\mathrm{cw}$ operation, ponderomotive forces produce a static frequency shift $\Delta f=$ $-k E_{\mathrm{acc}}{ }^{2}$, where $k$ is a function of the imbalance in the electric field caused by the cavity shape and the response of the cavity due to wall thickness and yield strength of the material. We want to calculate $k$ for the lowest natural vibration frequency of the stubs to determine the challenge for the frequency stabilization system. Consider a quarter wave resonator with a stub of length $L$ and rectangular cross section, $a=0.05 \mathrm{~m}$ by $b=0.03 \mathrm{~m}$, with the small side $b$, parallel to the beam axis $z$. The stub is fixed at one end and a force $F_{M}$ acts at the free end in the $z$ direction. The deflection of the end is given by $\Delta Z=F_{M} L^{3} /(3 E I)$, where $E=1.3 \times 10^{11} \mathrm{~Pa}$ is the modulus of elasticity of the copper and $I$ is the moment of inertia of the beam [9].

A typical coefficient of frequency detuning, $F_{T}$, of a $\beta_{o}=0.05,150 \mathrm{MHz}$ quarter wave cavity due to deflection a $\Delta Z$ of the drift tube is $F_{T}=2.5 \times 10^{8} \mathrm{~Hz} / \mathrm{m}$. The resultant frequency shift is given by $\Delta f=F_{T} \Delta Z$.

The coefficient of static frequency shift is then

$$
\begin{aligned}
k & =-\Delta f / E_{\mathrm{acc}}^{2}=-F_{T} \Delta Z / E_{\mathrm{acc}}^{2} \\
& =-F_{T} F_{M} L^{3} /\left(3 E I E_{\mathrm{acc}}{ }^{2}\right) .
\end{aligned}
$$

The eigenfrequency of oxygen-free copper beam of uniform cross section $A=a b$ deflecting parallel to the short side is given [10] by

$$
f_{\text {mech }}[\mathrm{Hz}]=2.04 \times 10^{3}(I / A)^{0.5} L^{-2},
$$

where all dimensions are in meters. By combining (3) and (4) and eliminating $I$, the equation for the static frequency shift $k$ for $E_{\text {acc }}=6.6 \mathrm{MV} / \mathrm{m}$ and $L=0.5 \mathrm{~m}$,

$$
\begin{aligned}
k & =-1.4 \times 10^{6} F_{T} F_{M} /\left(E E_{\mathrm{acc}}{ }^{2} A L f_{\text {mech }}{ }^{2}\right) \\
& \approx-8.1 \times 10^{4} F_{M} / f_{\text {mech }}{ }^{2}
\end{aligned}
$$

is obtained. 


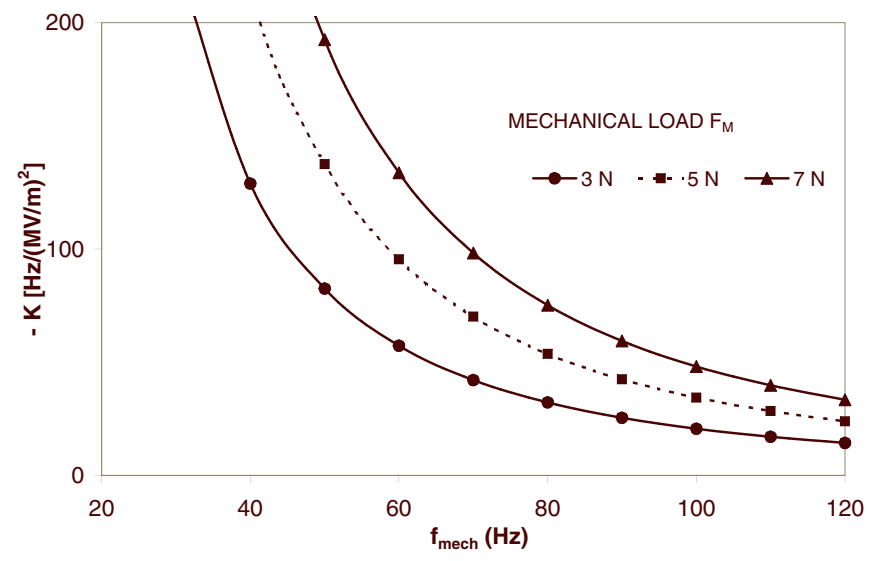

FIG. 7. Calculated rf frequency shift $k$ versus the frequency of the lowest mechanical mode $f_{\text {mech }}$ at different loading forces for a niobium on copper cavity at $E_{\text {acc }}=6.6 \mathrm{MV} / \mathrm{m}$.

The calculated $\mathrm{rf}$ frequency shift $k$ versus frequency for the lowest mechanical mode $f_{\text {mech }}$ at different loading forces $F_{M}$ in an $\mathrm{Nb}$ coated copper cavity is shown in Fig. 7.

As is evident from Fig. 7, an ideal goal for the lowest mechanical mode frequency is $F_{\text {mech }}>100 \mathrm{~Hz}$. First, high $F_{\text {mech }}$ minimizes coupling to low frequency ground and machinery vibrations and, second, $F_{\text {mech }}>100$ implies $k<50 \mathrm{~Hz} /(\mathrm{MV} / \mathrm{m})^{2}$.

\section{Window in the center stub to balance the electric force}

There is an imbalance of the electrical force on the outer loading elements of the 3-QWR because the $E$-field around them is intrinsically not symmetric. In contrast, the distribution of the $E$-field is reflection symmetric about the median plane of the inner stub. The higher electric field force between the outer loading elements and the inner one
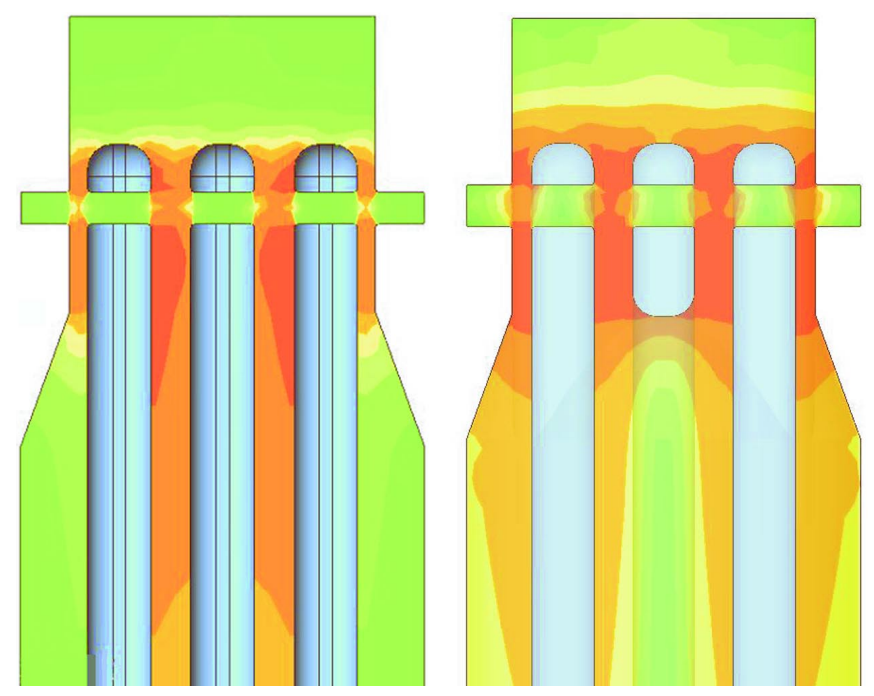

FIG. 8. (Color) The distribution of $E$-field in the 3-QWR with solid inner stub (left) and with the windowed one (right). can be reduced dramatically by introducing a window below the beam tube through the inner stub, thus reducing the area over which the electric field acts. The window does not extend to the base of the stub because the electric field is small there. Thus, a reduction in the area of the stub at the bottom results in little additional benefit. As well it is desirable to keep the cross section of the inner stub large in that location to minimize the current density. The distribution of $E$-field in the 3-QWR with a solid stub (left) and in a windowed one (right) is shown in Fig. 8. In the window design, the $E$-field on both sides of the outer stubs is approximately equal, minimizing the electric force imbalance and thus the ponderomotive effect.

\section{Mechanical modes}

Finite element structural analyses (STRAND7), was used to identify the six lowest mechanical modes which are shown in Fig. 9.

The first three are bending modes in which the stub ends move along the beam line: (i) mode 1, $59 \mathrm{~Hz}$ : only the drift tube of the inner element moves; (ii) mode 2, $63 \mathrm{~Hz}$ : the outer drift tubes both move in the same direction; (iii) mode 3, $64 \mathrm{~Hz}$ : the outer drift tubes move in opposite directions.

The second three are traverse-bending modes in which the drift tubes move in planes perpendicular to the beam axis: (i) mode 4, $95 \mathrm{~Hz}$ : the outer drift tubes both move in the same direction; (ii) mode 5, $100 \mathrm{~Hz}$ : the outer drift tubes move in opposite directions ; (iii) mode 6, $120 \mathrm{~Hz}$ : only the drift tube of the inner element moves.

The frequencies of modes $1,2,4$, and 6 are strongly affected by distortion of the shorting plate since the stubs themselves and the supporting column are quite stiff. The contribution of the shorting plate is minimal if its thickness is greater than $25 \mathrm{~mm}$. This is the case for the above frequency calculations.

The goal of having the lowest natural vibration frequency above $100 \mathrm{~Hz}$ is not achieved. The comparable lowest frequencies in successfully operating resonators are
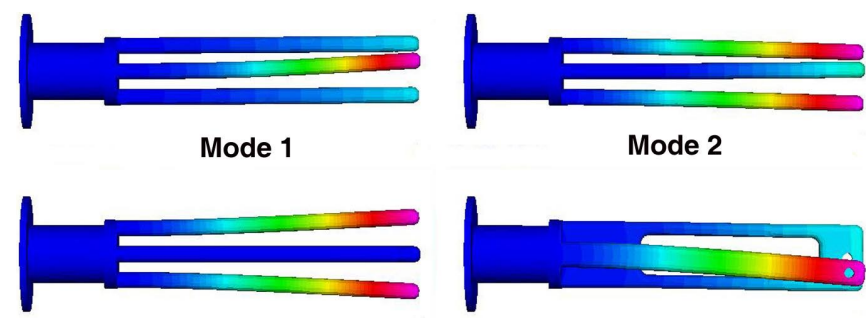

Mode 3

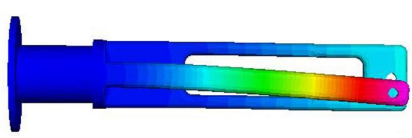

Mode 4
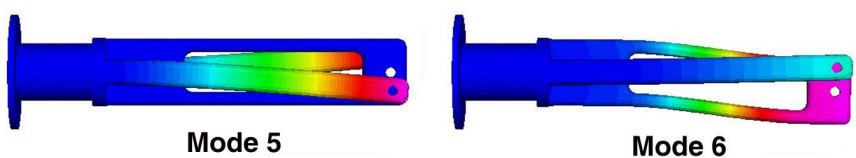

Mode 6

FIG. 9. (Color) The six lowest mechanical vibration modes in the 3-QWR. 


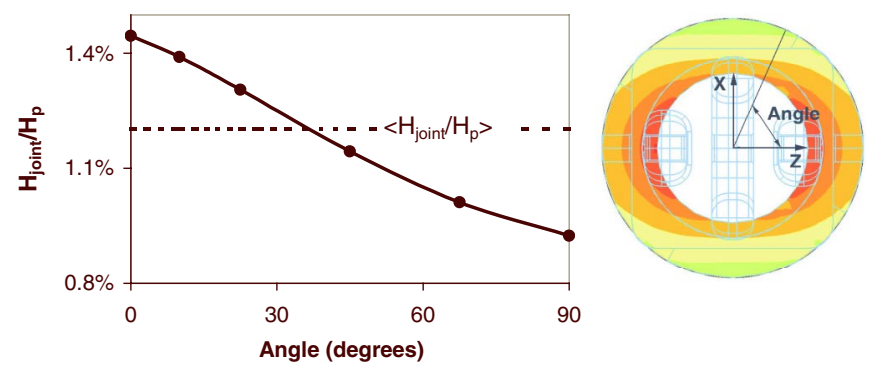

FIG. 10. (Color) The calculated ratio of the $H$-field to the peak magnetic field, $H_{p}$, in the shorting plate joint for the accelerating mode. The average value of ratio $H_{\text {joint }} / H_{p}$ is $1.2 \%$.

$\sim 50 \mathrm{~Hz}$ so the $59 \mathrm{~Hz}$ for the 3-QWR is probably high enough especially in a vibration-quiet laboratory.

\section{F. Demountable flange joints}

The 3-QWR employs two demountable flange joints. The ratio of the $H$-field in the shorting plate joint to the peak magnetic field of the resonator, $H_{\text {joint }} / H_{p}$, for the accelerating mode, is shown in Fig. 10. The attenuation of the rf current in the gasket in 3-QWR is nearly twice as effective as in the 2-QWR [2] since the mirror currents that flow in the outer cylinder travel over a $45^{\circ}$ segment in the $3-\mathrm{QWR}$ compared to $90^{\circ}$ in the 2-QWR. The magnetic field in the rf joint, at the edge of the shorting plate, $H_{\text {joint }} / H_{p}<1.5 \%$ for a column length of $157 \mathrm{~mm}$. $H_{\text {joint }}<$ $1.8 \mathrm{mT}$ at $H_{p}=120 \mathrm{mT}$ which corresponds to an accelerating field of $6.6 \mathrm{MV} / \mathrm{m}$ for $\mathrm{Nb}$ coated copper.

The $H$-field distributions in the outer cylinder for the nonoptimal modes 1 and 2 and the accelerating mode,

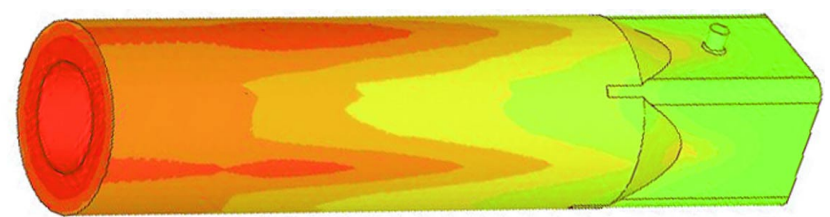

Mode 1
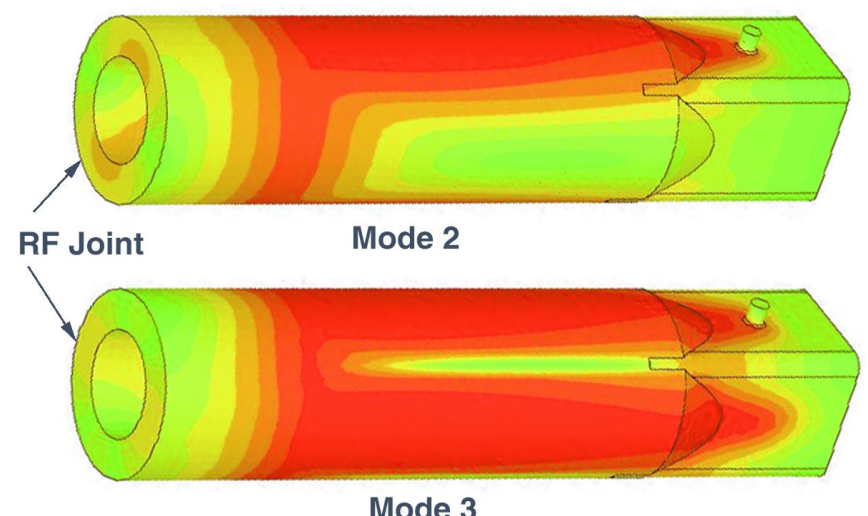

FIG. 11. (Color) Calculated $H$-fields for rf modes in the outer cylinder of the 3-QRW. mode 3, as calculated with MWS, are shown in Fig. 11. The currents going in the opposite directions in the outer conductor wall tend to connect in the wall. Thus, less current returns to the stub assembly through the joint as the supporting column is lengthened. It is this design feature of the 3-QWR that allows the minimization of $\mathrm{rf}$ current through the shorting plate joint.

As will be discussed in a subsequent publication, the current-minimizing feature of a supporting column can be exploited in mounting a displacement tuner in a 3-QWR.

The 3-QWR design minimizes the $H$-field in the gaskets for the accelerating mode 3 and reduces it in mode 2 . This is in marked contrast to the high rf current in the gasket for the quarter wave mode 1 .

\section{SUMMARY}

The 3-QWR provides 50\% more acceleration length as compare to 2-QWR because of the existence of an eigenmode that provides acceleration in each gap. Thus, for similar investments in electronics, cryogenics, and laboratory space, it is more efficient than an equivalent 2-QWR though the transit time effect reduces its efficiency by $\sim 2 \%$ and narrows its velocity acceptance by $\sim 28 \%$.

The MWS calculations for the 3-QWR indicate an acceptably low peak surface electric field ratio of $E_{p} / E_{\text {acc }}=$ 6.0. The peak magnetic field ratio is also low at $H_{p} / E_{\text {acc }}=$ $18.2 \mathrm{mT} / \mathrm{MV} / \mathrm{m}$.

The rf current in the gasket in 3-QWR is about one-half that in the 2-QWR. An Nb on copper 3-QWR, operating at its magnetic field limit, would have an accelerating field of $E_{\text {acc }}=6.6 \mathrm{MV} / \mathrm{m}$ and of $1.8 \mathrm{mT}$ in the gasket area. For the case of a $\mathrm{Pb}$ on $\mathrm{Cu} 3-\mathrm{QWR}$, the magnetic field limits the accelerating field to $E_{\mathrm{acc}}=2.9 \mathrm{MV} / \mathrm{m}$ when there would be $0.8 \mathrm{mT}$ in the gasket area. As in the case of the 2-QWR, these low magnetic fields in the joint locations allow the use of removable flanges and $\mathrm{rf}$ gaskets. Such flanges facilitate access to the interior of the resonator for manufacture, mechanical polishing, chemical surface treatment, high pressure rinsing, and coating with superconducting film.

The ponderomotive forces on the outer stubs can be balanced by the introduction of a window in the center stub.

The high stiffness of the 3-QWR provides mechanical stability against microphonics. The frequencies of the lowest mechanical modes are strongly affected by the distortion of the shorting plate but are above $59 \mathrm{~Hz}$ for a $25 \mathrm{~mm}$ thick plate. This, combined with low energy content, allows rf stabilization with a self-excited loop.

The 3-QWRs also have low currents between the outer conductor and the tuner flange. This allows the use of a displacement tuner mounted on a column instead of the conventional deflection plate.

Exploitation of a suite of 2-QWRs and 3-QWRs will extend the mass and energy ranges for the Heavy Ion 
Facility at the ANU. They can be also used for the very low energy sections of high current proton LINACs [11] or Heavy Ion Accelerators [12].

\section{ACKNOWLEDGMENTS}

The competence and skill of Alistair Muirhead in the use of Mechanical Desktop have been crucial for the project. We thank Benoit Gay for simulating mechanical properties of the resonator. The authors thank Computer-Simulation Technology $\mathrm{GmbH}$ for providing an evaluation license for package MWS to the Nuclear Physics Department and Dr. Frank Demming-Janssen, CST Headquarters, for his generous advise and cooperation during this work.

[1] G. P. Zinkann, in Proceedings of the 11th Workshop on RF Superconductivity, Travemunde, Germany (2003).

[2] N. R. Lobanov and D.C. Weisser, Phys. Rev. ST Accel. Beams 9, 042002 (2006).
[3] K. W. Shepard and P. N. Ostroumov, Phys. Rev. ST Accel. Beams 6, 080101 (2003).

[4] J. R. Delayen, Nucl. Instrum. Methods Phys. Res., Sect. A 259, 341 (1987).

[5] L. M. Bollinger, Annu. Rev. Nucl. Part. Sci. 36, 475 (1986).

[6] M.S. Mitrotzik and D. Prather, IEEE Spectrum 34, 53 (1997).

[7] J. R. Delayen, in Proceedings of the 10th Workshop on RF Superconductivity, KEK, Japan (2001), p. 152.

[8] D. C. Weisser and N. R. Lobanov, AIP Conf. Proc. 473, 117 (1999), edited by K. Shepard.

[9] E. Oberg and F.D. Jones, Machinery's Handbook (Industrial Press, England, 1968), 18th ed., p. 391.

[10] N. R. Lobanov and D. C. Weisser, in Proceedings of the 32nd Symposium of North Eastern Accelerator Personnel, edited by D. K. Hensley (World Scientific, Singapore, 1999), pp. 255-265.

[11] G. W. Foster, in Proceedings of the 12th Workshop on RF Superconductivity, Cornell, USA, 2005 (to be published).

[12] M.P. Kelly, in Proceedings of the 12th Workshop on RF Superconductivity, Cornell, USA, 2005 (to be published). 